\title{
Complicações e desfechos clínicos de pacientes em uso de nutrição parenteral em um hospital público do Distrito Federal
}

\author{
Complicacions and clinical outcomes of patients in use of parenteral nutrition in a public hos- \\ pital of the Federal District
}

DOI: $10.37111 /$ braspenj.2020353008

\author{
Mayra de Lima Granjeiro \\ Sheila Borges ${ }^{2}$ \\ Renata Costa Fortes ${ }^{3}$
}

\section{Unitermos:}

Desnutrição. Nutrição parenteral. Hiperglicemia.

\section{Keywords:}

Malnutrition. Parenteral nutrition. Hyperglycemia.

\section{Endereço para correspondência:}

Sheila Borges

Setor C Norte área especial 24 - Taguatinga, DF

Brasil - CEP 72120-970

E-mail: sbnutri12@hotmail.com

\section{Submissão}

23 de março de 2020

\section{Aceito para publicação}

8 de setembro de 2020

\begin{abstract}
RESUMO
Introdução: A nutrição parenteral tornou-se uma alternativa eficaz para oferta calórica-proteica em pacientes hospitalizados. O objetivo deste trabalho é avaliar a utilização, complicações e desfechos clínicos de pacientes em uso de nutrição parenteral. Método: Estudo analítico, quantitativo, longitudinal, retrospectivo com pacientes internados em um hospital público. As variáveis analisadas foram: sexo, idade, presença de desnutrição, risco nutricional, alcance de metas nutricionais, motivo de indicação, tipo de acesso, tempo de utilização, complicações presentes e óbitos. Resultados: A amostra foi constituída de 62 pacientes, com média de idade de $55,1 \pm$ 17,95 anos. A nutrição parenteral total foi encontrada em maior frequência $(61 \% ; n=38)$ e utilizada em até 30 dias $(74 \% ; n=28)$. Pela indicação, o pós-operatório teve prevalência em $45,2 \%$ $(n=28)$ dos casos. O risco nutricional $(58,1 \% ; n=36)$ e a desnutrição $(40,3 \% ; n=25)$ foram prevalentes. A hiperglicemia $(50 \% ; n=31)$ e a retenção hídrica $(48,4 \%, n=30)$ foram as complicações mais frequentes. A presença da retenção hídrica aumentou em 3,75 vezes as chances ao óbito $(\mathrm{OR}=3,75 ; 95 \% \mathrm{IC} 1,25-11,24 ; \mathrm{p}=0,018)$. Conclusão: $A$ indicação da nutrição parenteral total foi prevalente em situações de pós-operatório, em pacientes com risco nutricional e desnutrição. A hiperglicemia foi a complicação mais observada e a presença da retenção hídrica esteve associada a um maior risco de mortalidade no uso dessa terapia.
\end{abstract}

\section{ABSTRACT}

Introduction: Parenteral nutrition has become an effective alternative for caloric-protein supply in hospitalized patients. The objective of this study is to evaluate the use, complications and clinical outcomes of patients using parenteral nutrition. Methods: Analytical, quantitative, longitudinal, retrospective study with patients admitted to a public hospital. The variables analyzed were: sex, age, presence of malnutrition, nutritional risk, achievement of nutritional goals, reason for indication, type of access, time of use, present complications and deaths. Results: The sample consisted of 62 patients aged $55.1 \pm 17.95$ years old. Total parenteral nutrition was found more frequently $(61 \% ; n=38)$ and used within 30 days $(74 \% ; n=28)$. As indicated, the postoperative period was prevalent in $45.2 \%(n=28)$ of the cases. Nutritional risk $(58.1 \%, n=36)$ and malnutrition $(40.3 \%$; $n=25)$ were prevalent. Hyperglycemia $(50 \% ; n=31)$ and water retention $(48.4 \% ; n=30)$ were the most frequent complications. The presence of water retention increased the chances of death by 3.75 times (OR=3.75; 95\% IC 1.25-11.24; $p=0.018)$. Conclusion: The indication of parenteral nutrition was prevalent in postoperative situations, in patients with nutritional risk and malnutrition. Hyperglycemia was the most observed complication and the presence of water retention associated with a higher risk of mortality when using this therapy.

1. Nutricionista, Residente do Programa Multiprofissional em Saúde do Adulto e Idoso, Escola Superior de Ciências da Saúde, Fundação de Ensino e Pesquisa em Ciências da Saúde, Brasília, DF, Brasil.

2. Nutricionista, Mestranda em Ciências para a Saúde, Escola Superior de Ciências da Saúde, Fundação de Ensino e Pesquisa em Ciências da Saúde, Brasília, DF, Brasil.

3. Nutricionista, Doutora em Nutrição Humana pela Universidade de Brasília. Docente do Mestrado Profissional em Ciências para a Saúde da Escola Superior de Ciências da Saúde, Fundação de Ensino e Pesquisa em Ciências da Saúde, Brasília, DF, Brasil. 


\section{INTRODUÇ̃̃O}

A nutrição parenteral (NP) é utilizada preferencialmente em pacientes desnutridos ou em risco nutricional quando a nutrição enteral está contraindicada ou há intolerância, e também, quando não há função intestinal suficiente para manter ou restaurar o estado nutricional' .

A nutrição parenteral periférica (NPP) é administrada em veias periféricas, sendo uma via de complementação, não devendo ultrapassar o tempo limite de 14 dias de administração. Já a nutrição parenteral total (NPT) é realizada por meio de um acesso venoso central, podendo permanecer por um período maior e com osmolaridade acima do recomendado para NPP2.

Algumas complicações podem ocorrer durante o processo ou serem resultados dessa terapia nutricional e estão divididas em: mecânicas, infecciosas, metabólicas e nutricionais ${ }^{1,3}$. As principais intercorrências encontradas com uso da NP são: hiperglicemia; desidratação ou hiper-hidratação; aumento ou depleção de eletrólitos, como sódio, potássio, fósforo, magnésio e cálcio; hiperlipidemia, flebite, infecção de cateter e trombose ${ }^{4}$.

Pacientes desnutridos estão suscetíveis a maior risco de complicações. Estudos revelam que a prevalência de desnutrição hospitalar atualmente é elevada podendo ser maior que $60 \%$, levando a sérios problemas como aumento do tempo de internação, diminuição da qualidade de vida e aumento da mortalidade ${ }^{1,5}$.

O suporte nutricional adequado está associado à melhoria da sobrevida e à diminuição das taxas de readmissão hospitalar entre pacientes hospitalizados com desnutrição ${ }^{6}$. Em um estudo recente de coorte prospectivo envolvendo 147 pacientes, 15,6\% dos pacientes em NP foram a óbito, possivelmente devido à complexidade das doenças, sendo $81,4 \%$ com presença de desnutrição pela avaliação subjetiva global ${ }^{7}$.

A prescrição de NPT vem aumentando significativamente, eleva os gastos hospitalares e exige uma assessoria de profissionais obrigatórios por legislação: médico, nutricionista, farmacêutico e enfermeiro. É possível melhorar continuamente o cuidado nutricional e reduzir os custos com essa terapia nutricional por meio da implementação de protocolos e padronização de procedimentos ${ }^{8}$.

Conhecer o perfil nutricional anteriormente ao início da terapia permite um planejamento dietoterápico adequado, no intuito de corrigir os distúrbios nutricionais preexistentes e atender às demandas nutricionais. Adicionalmente, há a necessidade de se investigar as variáveis relacionadas a maior frequência de desnutrição e óbito, uma vez que, identificamse grupos mais suscetíveis a desfechos clínicos insatisfatórios?

Uma análise recente verificou que a terapia nutricional parenteral representa uma parte importante dos custos no tratamento de indivíduos hospitalizados, sendo o custo com essa terapia maior em grupo de usuários que foram a óbito ${ }^{10}$.
Nesse contexto, é necessário que os pacientes em NP sejam monitorados de acordo com os parâmetros nutricionais, infecciosos, mecânicos e metabólicos, para que ocorra uma redução nas complicações citadas. Vale ressaltar, quanto menor a incidência das complicações, maior será a chance de alcançar as metas nutricionais planejadas ${ }^{7}$.

O presente estudo teve como objetivo avaliar a utilização, as complicações presentes e os desfechos clínicos de pacientes em uso de NP em um hospital público do Distrito Federal.

\section{MÉTODO}

Tratou-se de um estudo analítico, quantitativo, longitudinal, retrospectivo, realizado no Hospital Regional de Taguatinga, com pacientes internados nas enfermarias de clínica médica, cirúrgica, ginecologia, cardiologia e unidade de terapia intensiva, no período de janeiro a agosto de 2018. A coleta de dados foi realizada por meio dos prontuários eletrônicos preenchidos por médicos e nutricionistas, com aplicação de um formulário próprio previamente elaborado. Os indivíduos elegíveis para o estudo foram todos os pacientes internados em uso de NP no período supracitado, seja como terapia nutricional exclusiva ou complementar, de ambos os sexos, com 18 anos ou mais de idade. Os pacientes em uso de NP em clínicas pediátricas e unidades de emergência foram excluídos desse estudo, bem como aqueles que receberam nutrição por via oral e/ou enteral exclusivas.

As variáveis analisadas foram: sexo, idade, presença de desnutrição, risco nutricional, alcance de metas calóricas e proteicas determinadas, motivo de indicação de NP, tipo de acesso, tempo de utilização, a presença das seguintes intercorrências: hiperglicemia (>140 mg/dL), hipoglicemia $(<70 \mathrm{mg} / \mathrm{dL})$, hipercalemia $(>5 \mathrm{mEq} / \mathrm{L})$, hipocalemia $(<3,6$ $\mathrm{mEq} / \mathrm{L})$, hipernatremia $(>148 \mathrm{mEq} / \mathrm{L})$, hiponatremia $(<135$ $\mathrm{mEq} / \mathrm{L})$, hipomagnesemia $(<1,8 \mathrm{mEq} / \mathrm{L})$, hiperfosfatemia $(>5,6 \mathrm{mg} / \mathrm{dL})$ e hipofosfatemia $(<2,5 \mathrm{mg} / \mathrm{L})$; hipertrigliceridemia (> $150 \mathrm{mg} / \mathrm{dL}$ ) e alteração hepática nos exames bioquímicos de dosagens das enzimas aspartato aminotransferase (AST $>38 \mathrm{UI} / \mathrm{L}$ ) e alanina aminotransferase (ALT > 48 $U \mathrm{U} / \mathrm{L}$ ). A presença de desidratação e retenção hídrica (sinal de cacifo/edema igual ou acima de $+/+4$ ) foi considerada por meio do exame físico no registro médico, sugerindo o acúmulo de líquido no espaço intersticial e expansão do volume extracelular. Também a ocorrência de flebite e infecção de cateter foram observadas em evoluções nos prontuários eletrônicos. Os desfechos clínicos de alta da NP ou óbito também foram avaliados.

Em relação ao risco nutricional, observou-se o resultado do instrumento aplicado de triagem Nutritional Risk Screening (NRS) ${ }^{11}$, que utiliza como parâmetros o índice de massa corporal, a gravidade da doença, a redução da ingestão alimentar, a perda de peso corporal e a idade. 
O diagnóstico nutricional de desnutrição foi classificado segundo a gravidade (grave ou não grave) e o contexto (doença ou trauma agudo, doença crônica, circunstâncias sociais e ambientais) pelo modelo da AND-ASPEN Clinical Characteristics Malnutrition ${ }^{12}$. Todos esses procedimentos e aplicação dessas ferramentas foram realizados pela equipe como atividades rotineiras dentro da atenção hospitalar, conforme protocolo do serviço.

As informações foram coletadas por meio da evolução médica e nutricional após o terceiro dia de uso da NP e os resultados dos exames bioquímicos comparados aos valores de referência do laboratório do próprio hospital.

Os dados foram agrupados em planilha do Microsoft Excel versão 2010 e analisados no programa estatístico IBM SPSS (Statistical Package for the Social Sciences) versão 23.0. A avaliação da normalidade de distribuição dos dados quantitativos foi feita por meio do teste Kolmogorov Smirnov. O teste t de Student foi empregado para amostras independentes e o teste Qui-quadrado de Pearson para variáveis categóricas. A probabilidade de significância considerada foi de $p<0,05$.

A análise de regressão logística múltipla foi empregada para verificar os fatores relacionados ao desfecho clínico de óbito. Para se obter um modelo satisfatório, as técnicas estatísticas de seleção das variáveis baseadas em inserção e na retirada das mesmas foram utilizadas (método forward stepwise/backward stepwise). Para melhores ajustes e poder preditivo, a razão de verossimilhança foi utilizada, a significância dos parâmetros da regressão foi analisada pelo teste estatístico de Wald e os testes de Omnibus e Hosmer e Lemeshow avaliaram a qualidade do modelo.

O presente estudo foi submetido e aprovado pelo Comitê de Ética e Pesquisa da Fundação de Ensino e Pesquisa em Ciências da Saúde da Secretaria de Estado de Saúde do Distrito Federal, número do parecer 3.253.223, Certificado de Apresentação para Apreciação Ética número 09398219.0.0000.5553.

\section{RESULTADOS}

Esse estudo teve como amostra 62 pacientes submetidos à NP no período avaliado, sendo $54,8 \%(n=34)$ do sexo masculino e $45,2 \%(n=28)$ do sexo feminino. Quanto à faixa etária, os pacientes possuíam média de idade de 55,15 1 17,95 anos.

○ tipo de terapia encontrada com maior frequência foi a NPT em $61 \%$ dos pacientes $(n=38)$, quanto à indicação mais frequente foi na situação de pós-operatório $(45,2 \%$, $\mathrm{n}=28)$ e utilização em até 30 dias $(74 \%, \mathrm{n}=28)$ para NPT. Já para NPP, 96\% ( $n=23)$ utilizaram por menos de 10 dias (Tabela 1). A clínica com maior presença do uso da NP foi a cirúrgica, em $56 \%(n=35)$ dos participantes, seguida da unidade de terapia intensiva $(29 \% ; n=18)$.
Tabela 1 - Análise descritiva dos participantes recebendo nutrição parenteral internados em um hospital público.

\begin{tabular}{|c|c|c|}
\hline \multirow[t]{2}{*}{ Variável } & \multicolumn{2}{|c|}{ Frequência } \\
\hline & $\mathrm{n}$ & $\%$ \\
\hline \multicolumn{3}{|l|}{ Sexo } \\
\hline Masculino & 34 & 54,8 \\
\hline Feminino & 28 & 45,2 \\
\hline \multicolumn{3}{|l|}{ Via de nutrição parenteral } \\
\hline Nutrição parenteral periférica & 24 & 39,0 \\
\hline Nutrição parenteral total & 38 & 61,0 \\
\hline \multicolumn{3}{|l|}{ Motivos de indicação } \\
\hline Câncer & 12 & 19,3 \\
\hline Doenças do trato gastrointestinal & 14 & 22,6 \\
\hline Pós-operatório & 28 & 45,2 \\
\hline Doenças neurológicas & 3 & 4,8 \\
\hline Outras & 5 & 8,1 \\
\hline \multicolumn{3}{|c|}{ Tempo de utilização de nutrição parenteral total } \\
\hline Até 30 dias & 28 & 74,0 \\
\hline 1 a 3 meses & 7 & 18,0 \\
\hline 3 a 6 meses & 2 & 5,0 \\
\hline Acima de 6 meses & 1 & 3,0 \\
\hline \multicolumn{3}{|c|}{ Tempo de utilização de nutrição parenteral periférica } \\
\hline Menos de 10 dias & 23 & 96,0 \\
\hline Mais de 10 dias & 1 & 4,0 \\
\hline \multicolumn{3}{|l|}{ Clínica/Unidade hospitalar } \\
\hline Cirúrgica & 35 & 56,0 \\
\hline Unidade de terapia intensiva & 18 & 29,0 \\
\hline Clínica médica & 7 & 11,0 \\
\hline Ginecologia & 1 & 2,0 \\
\hline Cardiologia & 1 & 2,0 \\
\hline \multicolumn{3}{|c|}{ Ocorrência de flebite em nutrição parenteral periférica } \\
\hline $\operatorname{Sim}$ & 1 & 4,0 \\
\hline Não & 23 & 96,0 \\
\hline \multicolumn{3}{|l|}{ Infecção de cateter } \\
\hline $\operatorname{Sim}$ & 5 & 8,0 \\
\hline Não & 56 & 90,4 \\
\hline Ausente $^{*}$ & 1 & 1,6 \\
\hline \multicolumn{3}{|c|}{ Metas calóricas e proteicas alcançadas } \\
\hline Sim & 31 & 50,0 \\
\hline Não & 6 & 9,7 \\
\hline Ausente* $^{*}$ & 25 & 40,3 \\
\hline \multicolumn{3}{|l|}{ Risco nutricional } \\
\hline Sim & 36 & 58,1 \\
\hline Não & 3 & 4,8 \\
\hline Ausente & 23 & 37,1 \\
\hline \multicolumn{3}{|l|}{ Desnutrição } \\
\hline Sim & 25 & 40,3 \\
\hline Não & 6 & 9,7 \\
\hline Ausente* $^{\star}$ & 31 & 50,0 \\
\hline \multicolumn{3}{|l|}{ Desfecho clínico } \\
\hline Óbito & 26 & 42,0 \\
\hline Alta da nutrição parenteral & 36 & 58,0 \\
\hline Total & 62 & 100,0 \\
\hline
\end{tabular}


Em relação à NPP, apenas um (4\%) paciente apresentou ocorrência de flebite dentre aqueles que utilizaram essa terapia $(n=24)$. A maioria dos participantes $(90,4 \%, n=56)$ não apresentou registro de infecção de acesso da NP. Essa informação não foi encontrada no prontuário de apenas um participante.

Do total de participantes, $50 \%(n=31)$ tiveram ambas metas nutricionais atingidas pela NP e a maioria dos participantes $(58,1 \% ; n=36)$ apresentou risco nutricional (Tabela 1).

Pelo diagnóstico nutricional, metade da amostra $(n=31)$ não apresentov essa informação no prontuário. Dos demais, 40,3\% ( $n=25)$ dos pacientes apresentaram desnutrição pelos critérios da AND-ASPEN Clinical Characteristics Malnutrition ${ }^{12}$. Do total, $42 \%(n=26)$ foram a óbito.

As principais complicações encontradas foram hiperglicemia $(50 \%, n=31)$, retenção hídrica $(48,4 \%, n=30)$ e alteração hepática $(45,0 \%, n=28)$, porém, houve ausência de informações nos prontuários sobre hipertrigliceridemia, hiperfosfatemia, hipofosfatemia e hipomagnesemia. A desidratação foi encontrada em 29,1\% (n=18) dos participantes (Figura 1).
As complicações encontradas de hipoglicemia, hipercalemia, hipernatremia e hiponatremia foram com menores frequências: $1,6 \%(n=1) ; 4,8 \%(n=3) ; 12,9 \%(n=8) ; 14,3 \%$ $(n=9)$, respectivamente.

As variáveis qualitativas de sexo, complicações categorizadas em presentes ou ausentes, metas nutricionais atingidas, presença de risco nutricional e de desnutrição foram associadas ao desfecho clínicos dos participantes que receberam NP (Tabela 2). Houve diferença estatística para as variáveis de retenção hídrica $(p=0,007)$ e hipomagnesemia $(p=0,005)$.

A regressão logística binária múltipla identificou as principais variáveis que poderiam explicar o desfecho clínico de óbito. Devido à ausência de alguns parâmetros em prontuários, as variáveis com pelo menos 50 dados registrados em prontuários foram inseridas e os resultados foram avaliados, a fim de melhorar a previsão do modelo de regressão. As variáveis com quantidade menor foram excluídas do modelo, pois prejudicariam o ajuste do mesmo (Tabela 3).

As variáveis independentes selecionadas de acordo com a melhor adequação para o modelo de regressão foram:

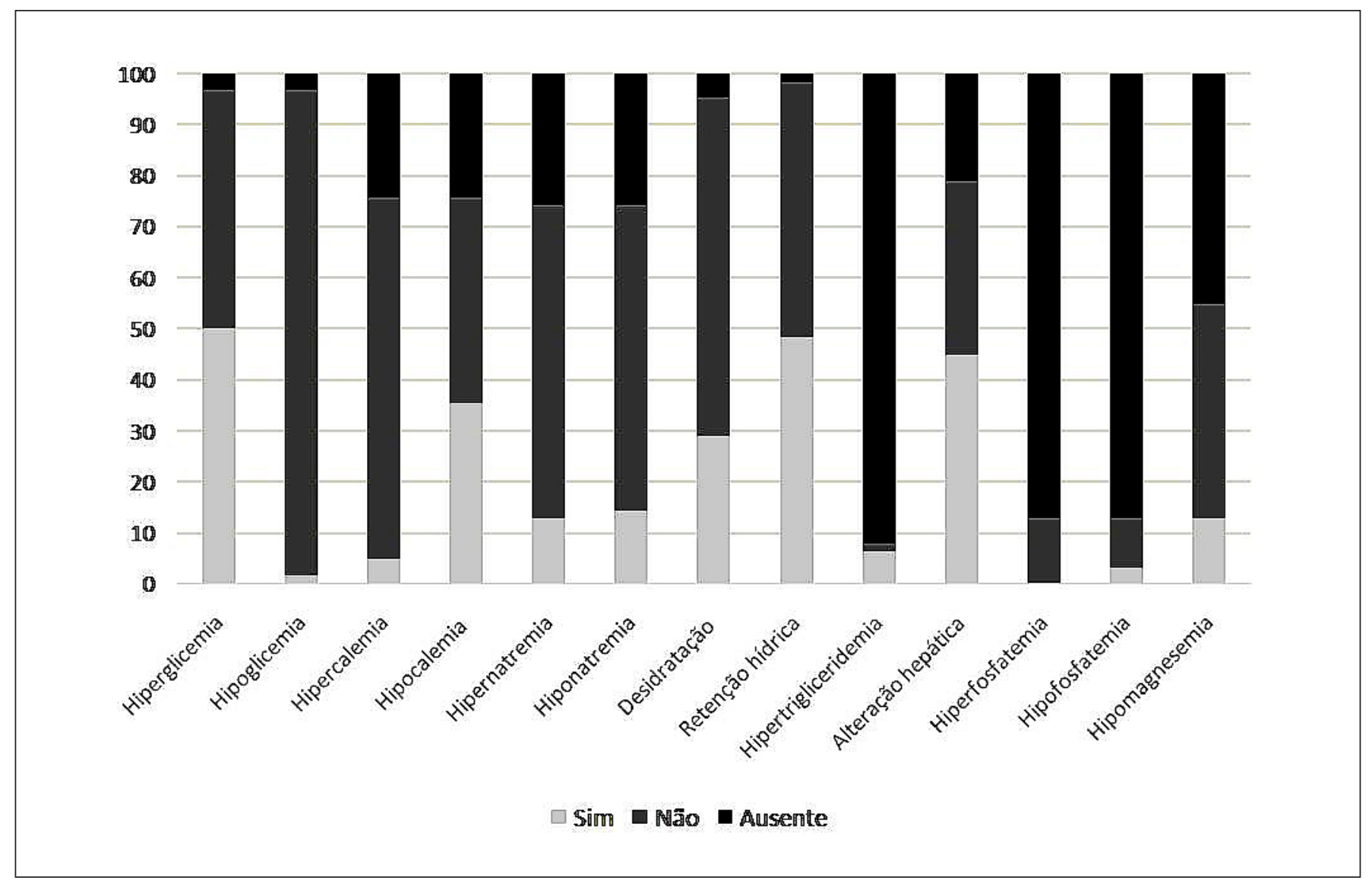

Figura 1 - Frequência das complicações encontradas dos participantes recebendo nutrição parenteral internados em um hospital público.

*Ausente: falta de informações nos prontuários. 
Tabela 2 - Análise das variáveis em relação aos desfechos clínicos (alta da nutrição parenteral e óbitos) dos participantes recebendo nutrição parenteral internados em um hospital público.

\begin{tabular}{|c|c|c|c|}
\hline & \multicolumn{2}{|c|}{ Desfechos clínicos } & \multirow[b]{3}{*}{$p$-valor } \\
\hline & Óbitos & Alta da NP** & \\
\hline & $\mathrm{n}(\%)$ & $\mathrm{n}(\%)$ & \\
\hline Sexo & & & 0,243 \\
\hline Masculino & $12(19,3)$ & $22(35,5)$ & \\
\hline Feminino & $14(22,6)$ & $14(22,6)$ & \\
\hline Hiperglicemia & & & 0,832 \\
\hline Sim & $19(30,7)$ & $12(19,3)$ & \\
\hline Não & $7(11,3)$ & $22(35,5)$ & \\
\hline Ausente* & _ & $2(3,2)$ & \\
\hline Hipoglicemia & & & 0,892 \\
\hline $\operatorname{Sim}$ & $1(1,6)$ & - & \\
\hline Não & $25(40,3)$ & $34(54,9)$ & \\
\hline Ausente* $^{*}$ & - & $2(3,2)$ & \\
\hline Hipercalemia & & & 0,190 \\
\hline Sim & $3(4,8)$ & - & \\
\hline Não & $19(30,7)$ & $25(40,3)$ & \\
\hline Ausente* & $4(6,4)$ & $11(17,8)$ & \\
\hline Hipocalemia & & & 0,447 \\
\hline $\operatorname{Sim}$ & $9(14,5)$ & $13(21,0)$ & \\
\hline Não & $13(21,0)$ & $12(19,3)$ & \\
\hline Ausente* & $4(6,4)$ & $11(17,8)$ & \\
\hline Hipernatremia & & & 0,508 \\
\hline Sim & $5(8,1)$ & $3(4,8)$ & \\
\hline Não & $16(25,7)$ & $22(35,5)$ & \\
\hline Ausente* & $5(8,1)$ & $11(17,8)$ & \\
\hline Hiponatremia & & & 0,650 \\
\hline Sim & $3(4,8)$ & $6(9,5)$ & \\
\hline Não & $18(29,1)$ & $19(30,7)$ & \\
\hline Ausente* & $5(8,1)$ & $11(17,8)$ & \\
\hline Desidratação & & & 0,239 \\
\hline Sim & $10(16,2)$ & $8(12,9)$ & \\
\hline Não & $16(25,8)$ & $25(40,3)$ & \\
\hline Ausente* $^{*}$ & 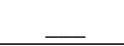 & $3(4,8)$ & \\
\hline Retenção hídrica & & & 0,007 \\
\hline Sim & $18(29,1)$ & $12(19,3)$ & \\
\hline Não & $8(12,9)$ & $23(37,1)$ & \\
\hline Ausente* & 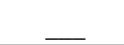 & $1(1,6)$ & \\
\hline Hipertrigliceridemia & & & 0,819 \\
\hline Sim & $3(4,8)$ & $1(1,6)$ & \\
\hline Não & - & $1(1,6)$ & \\
\hline Ausente* & $23(37,1)$ & $34(54,9)$ & \\
\hline Alteração hepática & & & 0,934 \\
\hline Sim & $13(21,0)$ & $15(24,0)$ & \\
\hline Não & $10(16,2)$ & $11(17,8)$ & \\
\hline Ausente* & $3(4,8)$ & $10(16,2)$ & \\
\hline Hiperfosfatemia & & & - \\
\hline Sim & - & - & \\
\hline Não & $4(6,4)$ & $4(6,4)$ & \\
\hline Ausente* & $22(35,5)$ & $32(51,7)$ & \\
\hline
\end{tabular}

Continuação Tabela 2 - Análise das variáveis em relação aos desfechos clínicos (alta da nutrição parenteral e óbitos) dos participantes recebendo nutrição parenteral internados em um hospital público.

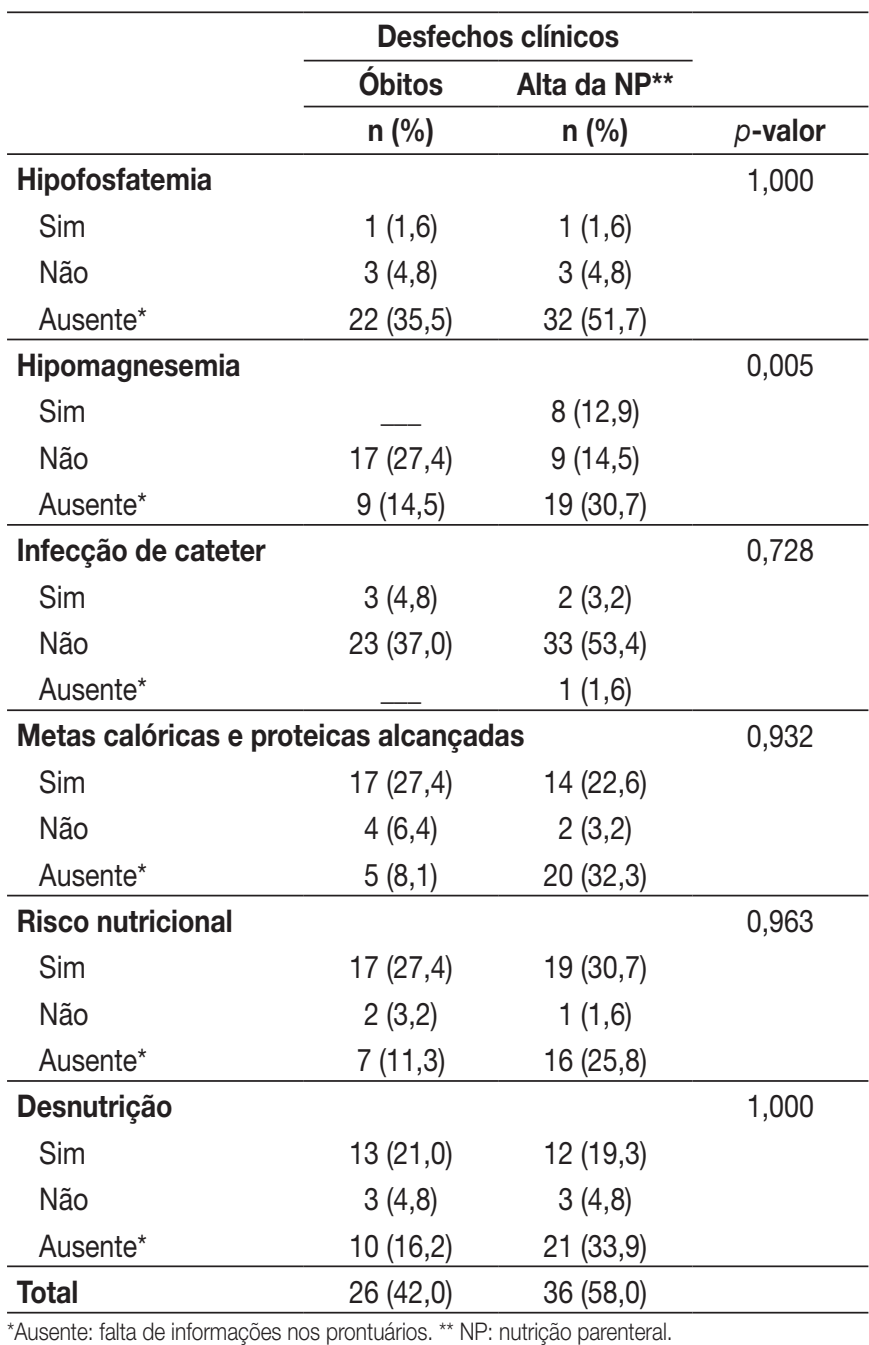

sexo, idade, hiperglicemia, desidratação, retenção hídrica e infecção de cateter. $\bigcirc$ poder preditivo foi de $70,7 \%$ para os desfechos clínicos (óbitos) dos participantes do estudo. $\bigcirc$ modelo foi estatisticamente significativo $(p=0,013)$ para o teste de Omnibus e obteve um valor satisfatório para o teste de Hosmer e Lemeshow $(p=0,158)$, ou seja, não se rejeita a hipótese nula no estudo.

Apenas a variável retenção hídrica apresentou significância estatística $(p=0,018)$. A técnica de seleção de variáveis utilizada na regressão logística comprovou o modelo com melhor ajuste após uma etapa de inserção de variáveis, apenas com a retenção hídrica (Tabela 4). Confirmou-se, nesse estudo, essa variável ser a principal relacionada à mortalidade. Pacientes em NP com retenção hídrica apresentaram 3,75 vezes mais chance de ir a óbito quando comparados àqueles sem retenção $(O R=3,75 ; 95 \% \mid C 1,25$ $11,24 ; p=0,018)$. 
Tabela 3 - Regressão logística para a variável de desfecho clínico de óbito dos participantes recebendo nutrição parenteral internados em um hospital público.

\begin{tabular}{lccc}
\hline Variável & p-valor & OR $^{*}$ & 95\% IC** \\
\hline Sexo & 0,092 & 0,33 & $0,09-1,20$ \\
Idade & 0,105 & 0,96 & $0,92-1,39$ \\
Hiperglicemia & 0,403 & 0,51 & $0,11-2,46$ \\
Desidratação & 0,117 & 3,13 & $0,75-13,08$ \\
Retenção hídrica & 0,018 & 4,50 & $1,29-15,70$ \\
Infecção de cateter & 0,420 & 2,39 & $0,29-19,71$ \\
\hline
\end{tabular}

*OR: odds ratio/risco de chance para o óbito; ${ }^{* \star} 95 \%$ IC: intervalo de $95 \%$ de confiança para a razão de risco.

Tabela 4 - Regressão logística com seleção de variáveis para desfecho clínico de óbito dos participantes recebendo nutrição parenteral internados em um hospital público.

\begin{tabular}{lccc}
\hline Variável & $\mathrm{p}$-valor & OR $^{*}$ & 95\% IC \\
\hline Retenção hídrica & 0,018 & 3,75 & $1,25-11,24$
\end{tabular}

*OR: odds ratio/risco de chance para o óbito; ${ }^{* \star} 95 \%$ IC: intervalo de $95 \%$ de confiança para a razão de risco.

\section{DISCUSSÃO}

Neste estudo, as indicações de NP em pós-operatório $(45,2 \% ; n=28)$ foram mais prevalentes. O estudo de Mazzaro et al. ${ }^{13}$, também conduzido em um hospital público do Distrito Federal, sobre o perfil de 63 pacientes em uso de NP, evidenciou as seguintes indicações da terapia: câncer $(50,8 \%)$, pacientes cirúrgicos $(25,4 \%)$ e doenças do trato gastrointestinal (6,3\%). Um estudo realizado em um hospital situado na Índia, a média de idade dos participantes foi de $54 \pm 14,8$ anos e as cirurgias do trato gastrointestinal foram mais prevalentes na indicação da NP $(55,6 \% ; n=30)^{14}$.

Observou-se a prevalência da utilização mais prolongada da NPT em até 30 dias (74\%; $n=28)$, sem registros de infecções de acesso em 90,4\% $(n=56)$ do total da amostra. Em um estudo realizado em um hospital municipal de Teresina sobre a avaliação de 313 prescrições de NP, ocorrência do uso dessa terapia foi também prevalente na clínica cirúrgica $(60,61 \% ; n=83)$, a NPT foi prescrita em $87,86 \%$ da amostra e apenas $8,7 \%$ utilizaram a NP por mais de 15 dias $^{15}$. De forma semelhante, Parra-Flores et al. ${ }^{16}$ verificaram ausência de infecção em $81 \%(n=69)$ dos participantes e um período aproximadamente de 34 dias com acesso venoso central sem essa intercorrência.

Pela análise dos participantes em NPP, 96\% $(n=23)$ possuíram indicação por menos de 10 dias, esse achado se aproxima da recomendação do uso dessa terapia por esse período, em virtude das formulações parenterais periféricas poderem causar flebite. Constatou-se apenas um paciente com essa intercorrência. Sugrue et al. ${ }^{17}$, de forma discrepante, conduziram uma pesquisa retrospectiva com 159 pacientes em uso de NPP, observaram cerca de 43,4\% $(n=69) \mathrm{com}$ flebite, considerando um tempo apropriado máximo de 14 dias para utilização da NPP.

Em relação às complicações metabólicas, resultados adversos estão associados à hiperglicemia em pacientes que recebem NPT, como, por exemplo, alterações cardiovasculares e mortalidade. Os fatores de risco que também podem contribuir para o desenvolvimento de hiperglicemia em resposta à NPT na ausência de diabetes incluem: idade, quantidade de dextrose administrada, infecção, gravidade da doença e a interação desses fatores ${ }^{7}$.

Neste estudo, $50 \%(n=31)$ dos participantes apresentaram hiperglicemia, contudo, como limitação deste estudo, não houve separação de pacientes com diabetes em uso de NP. A American Society for Parenteral and Enteral Nutrition (ASPEN) ${ }^{18}$ considera para pacientes adultos hospitalizados a hiperglicemia maior que 140 a 180 mg/dL. A prevalência de hiperglicemia é bastante alta em pacientes não críticos, afetando o tratamento com insulina e o controle metabólico. Um grupo de estudos sobre hiperglicemia em NP analisou a glicemia de pacientes submetidos à NPT, cerca de metade dos indivíduos analisados $(56,6 \%)$ tinha pelo menos uma glicemia capilar maior que $180 \mathrm{mg} / \mathrm{dL}^{19}$, índices semelhantes aos encontrados no presente estudo. Olveira et al. ${ }^{20}$ verificaram que, em 605 pacientes não críticos com níveis séricos de glicose maior que $180 \mathrm{mg} / \mathrm{dL}$ durante a infusão de NPT tiveram 5,6 vezes mais chances de óbito do que aqueles com níveis séricos de glicose menor que $140 \mathrm{mg} / \mathrm{dL}$.

Já a hipocalemia ocorrida com o uso da NP (35,5\%, $n=22$ ), outra alteração metabólica encontrada neste estudo, possivelmente, pode ser causada por alcalose metabólica ou por medicamentos, como diuréticos, mineralocorticoides, aminoglicosídeos e anfotericina ${ }^{4}$. Em uma pesquisa prospectiva de acompanhamento de 80 pacientes em NPT por sete dias, realizada por Pantoja et al. ${ }^{21}$, 27,5\% dos pacientes diagnosticados com alto risco para síndrome da realimentação tiveram hipocalemia. $O$ suporte parenteral é um fator de risco para essa síndrome e complicações metabólicas, como hipofosfatemia, hipomagnesemia e hipocalemia, são comumente encontradas nessa situação ${ }^{4}$. No entanto, esses eletrólitos não estavam com os resultados disponíveis na maioria dos prontuários, o que dificultou a identificação da síndrome de realimentação e sua relação com desfechos de óbitos.

Para a hipertrigliceridemia, no presente estudo, considerou-se níveis plasmáticos de triglicerídeos maiores que $150 \mathrm{mg} / \mathrm{dL}$, conforme referência do laboratório do hospital. A maioria dos participantes $(92,0 \%, n=57)$ não apresentava resultados dessa intercorrência metabólica 
em prontuários, encontrada apenas em quatro dos participantes. Em um estudo de coorte observacional retrospectivo envolvendo 110 pacientes recebendo NPT, a incidência de hipertrigliceridemia, considerada acima de $200 \mathrm{mg} /$ $\mathrm{dL}$, foi de $33,3 \%^{22}$. Nesse estudo, os autores justificam o aparecimento dessa complicação metabólica à condição clínica do paciente ou à sobrecarga de carboidratos na NPT. Reforça-se a importância da dosagem dos triglicerídeos em pacientes recebendo NP, pelo fato de que a infusão venosa em doses elevadas desses macronutrientes pode interferir nos valores séricos e contribuir para a sobrecarga retículoendotetial hepática, o que gera alterações nas dosagens de AST e ALT (enzimas hepáticas). A hipertrigliceridemia é um fator de risco para pancreatite em NPT. Em relação à alteração hepática (definida neste estudo como elevação das transaminases após $03^{\circ}$ dia de administração da $N P)$, foi encontrada prevalência de $45 \%(n=28)$ dos participantes. Do mesmo modo, Lakananurak e Tienchai ${ }^{23}$ evidenciaram prevalência dessa complicação em 59,1\% $(n=26)$ dos pacientes recebendo NP. Comumente, ocorre em pacientes pós-operatórios ou críticos devido à lesão hepática hipóxica ou hepatite isquêmica ${ }^{4}$. Llop-Talaveron et al. ${ }^{24}$ verificaram que, após 8 dias de administração de NP, em 181 pacientes, houve também disfunção hepática por elevação das transaminases.

A retenção hídrica esteve presente em 48,4\% $(n=30)$ dos participantes em uso de NP neste estudo, porém pode não estar atribuída exclusivamente a essa terapia nutricional, uma vez que, em situações de pós-operatório ou em pacientes críticos, ocorrem baixas concentrações agudas de albumina, possivelmente levando à presença de edema. Um fator que poderia também contribuir para a ocorrência dessa complicação seria a hidratação venosa em pós-operatório em níveis acima de $30 \mathrm{ml} / \mathrm{kg} \mathrm{em} 24$ horas $^{4}$. A maior parte dos participantes $(45,2 \% ; n=28)$ envolvidos neste estudo estava em condições de pós-operatório.

Em relação à avaliação nutricional, 37,1\% $(n=23)$ dos pacientes não continham informação sobre risco nutricional, metade $(50 \% ; n=31)$ não tinha informações sobre o diagnóstico nutricional em prontuários e sobre as metas nutricionais alcançadas, $40,4 \%(n=25)$ dos prontuários dos pacientes não continham esse registro. Esses achados foram encontrados em não conformidade em comparação aos indicadores de qualidade em terapia nutricional preconizados pelo International Life Sciences Institute (ILSI) do Brasil (2018) ${ }^{25}$. As metas recomendadas para esses indicadores são: para frequência de realização da triagem nutricional é de maior ou igual a $90 \%$ dos pacientes hospitalizados; para frequência de realização do diagnóstico nutricional e do alcance das necessidades calóricas e proteicas é de maior ou igual a $80 \%$ para esses ambos indicadores.
A prevalência de desnutrição foi de 40,3\% ( $n=25)$ na subamostra de 31 participantes que continha essa informação no prontuário. Segundo a literatura científica, a presença desse distúrbio nutricional em ambiente hospitalar pode chegar a índices maiores que 60\%, podendo ocasionar agravos hospitalares, como aumento do tempo de internação, maior risco de complicações, infecções, diminuição da qualidade de vida e aumento da mortalidade ${ }^{6}$. Contudo, um fator limitante nesse estudo é a ausência de associação entre a presença do risco nutricional e a desnutrição com os desfechos clínicos de óbitos pela ausência dessas informações na amostra avaliada.

Por meio da análise de regressão logística, observou-se que pacientes com retenção hídrica (edema) em uso de NP apresentaram 3,75 vezes mais chances de vir a óbito em comparação aos doentes sem essa condição. $\bigcirc$ aumento anormal de líquido extracelular geralmente ocorre em pacientes com insuficiência renal ou em administração de doses excessivas de NP ou pela hidratação venosa ${ }^{4}$. Pacientes com doenças cardíacas e hepáticas, concomitantemente, também podem influenciar no aumento de líquido extracelular, ocasionando sintomas, como taquipneia, ganho agudo de peso e hipoalbuminemia ${ }^{24}$.

A hiponatremia pode revelar a retenção hídrica, no entanto, nessa pesquisa, 59,8\% $(n=37)$ do total de 46 participantes que tinham registro dessa complicação não tiveram essa alteração metabólica. Já a concentração sérica de magnésio é a única medida prática de avaliação desse eletrólito, mas não é a melhor indicação, pela maior parte desse mineral no organismo estar localizada intracelularmente e no osso. Nesse estudo, encontrou-se 12,9\% (n=8) de hipomagnesemia em 34 pacientes que continham esse resultado, com diferença estatística significativa para essa variável $(p=0,005)$, contudo, não foi possível obter a razão de chance na análise de regressão logística em relação ao desfecho clínico de óbito.

É importante salientar que uma das complicações com menos dados ausentes em prontuários foi a retenção hídrica. Sendo assim, pode-se inferir que outras variáveis poderiam também estar associadas à mortalidade na amostra, caso tivessem informações completas para realização da análise dos dados.

Segundo Worthington et al. ${ }^{1}$, durante o suporte nutricional parenteral, deve-se monitorar periodicamente glicose, creatinina, eletrólitos, cálcio, magnésio, fósforo, hemograma completo, bilirrubina total e indireta, fosfatase alcalina, transaminases, tempo de protrombina, tempo de tromboplastina parcial, triglicerídeos e proteínas séricas em pacientes hospitalizados. Esse estudo revela a fragilidade da assistência hospitalar pela ausência de informações importantes para o acompanhamento do paciente em terapia nutricional parenteral. 


\section{CONCLUSÃO}

No presente estudo, observou-se uma prevalência do uso de NPT em situações de pós-operatório, administrada pelo período de até 30 dias, em pacientes com risco nutricional e desnutrição predominantemente.

A hiperglicemia foi a complicação metabólica mais frequente. A retenção hídrica esteve associada à mortalidade em pacientes em uso de terapia nutricional parenteral, aumentando em 3,75 vezes as chances de óbito.

Evidencia-se a necessidade de uma maior atenção da equipe multiprofissional de terapia nutricional na evolução e no monitoramento da NP. O acompanhamento da utilização dessa terapia é primordial para o planejamento das atividades do setor hospitalar, buscando a minimização de complicações e desfechos clínicos desfavoráveis.

\section{REFERÊNCIAS}

1. Worthington P, Balint J, Bechtold M, Bingham A, Chan LN, Durfee S, et al. When is parenteral nutrition appropriate? JPEN J Parenter Enteral Nutr. 2017;41(3):324-77.

2. Pittiruti M, Hamilton H, Biffi R, MacFie J, Pertkiewicz M; ESPEN. ESPEN Guidelines on Parenteral Nutrition: central venous catheters (access, care, diagnosis and therapy of complications). Clin Nutr. 2009;28(4):365-77.

3. Ayers P, Boullata J, Sacks G. Parenteral nutrition safety: the story continues. Nutr Clin Pract. 2018;33(1):46-52.

4. Lappas BM, Patel D, Kumpf V, Adams DW, Seidner DL. Parenteral nutrition: indications, access, and complications. Gastroenterol Clin North Am. 2018;47(1):39-59.

5. Eglseer D, Halfens RJ, Lohrmann C. Is the presence of a validated malnutrition screening tool associated with better nutritional care in hospitalized patients? Nutrition. 2017;37:104-11.

6. Gomes F, Baumgartner A, Bounoure L, Bally M, Nicolas ED, Greenwald JL, et al. Association of nutritional support with clinical outcomes among medical inpatients who are malnourished or at nutritional risk: an updated systematic review and meta-analysis. JAMA Netw Open. 2019;2(11):e1-1915138.

7. Adjemian D, Arendt BM, Allard JP. Assessment of parenteral nutrition prescription in Canadian acute care settings. Nutrition. 2018;49:7-12.

8. van Schaik R, Van den Abeele KV, Melsens G, Schepens P, Lanssens T, Vlaemynck B, et al. A protocol for sustained reduction of total parenteral nutrition and cost savings by improvement of nutritional care in hospitals. Clin Nutr ESPEN. 2016;15:114-21.

9. Santos CA, Firmino HH, Esmeraldo MLF, Alfenas RCG, Rosa $\mathrm{COB}$, Ribeiro AQ, et al. Perfil nutricional e fatores associados à desnutrição e ao óbito em pacientes com indicação de terapia nutricional. BRASPEN J. 2017;32(1):30-5.

10. Hyeda A, Costa ESM. Análise econômica dos custos com terapia nutricional enteral e parenteral conforme doença e desfecho. Einstein. 2017;15(2):192-9.

11. Kondrup J, Rasmussen HH, Hamberg O, Stanga Z; Ad Hoc ESPEN Working Group. Nutritional risk screening (NRS 2002): a new method based on an analysis of controlled clinical trial. Clin Nutr. 2003;22(3):321-36.

12. White JV, Guenter P, Jensen G, Malone A, Schofield M; Academy Malnutrition Work Group; A.S.P.E.N. Malnutrition Task Force;
A.S.P.E.N. Board of Directors. Consensus statement: Academy of Nutrition and Dietetics and American Society for Parenteral and Enteral Nutrition: characteristics recommended for the identification and documentation of adult malnutrition (undernutrition). JPEN J Parenter Enteral Nutr. 2012;36(3):275-83.

13. Mazzaro AL, Coelho MS, Souza BAT, Ceniccola GD. Perfil de pacientes em nutrição parenteral e a influência do estado nutricional no tempo de acompanhamento da equipe multiprofissional de terapia nutricional. BRASPEN J. 2019;34(3):287-92.

14. Ramakrishnan N, Shankar B, Ranganathan L, Daphnee DK, Bharadwaj A, Venkataraman R. Parenteral nutrition support: beyond gut feeling? Quality control study of parenteral nutrition practices in a tertiary care hospital. Indian J Crit Care Med. 2016;20(1):36-9.

15. Coelho ML, Barros YSO, Bráulio CA, Barros IC, Brandão MS, Carneiro SMP. Terapia nutricional parenteral: avaliação da prescrição, composição e aspectos econômicos em um hospital de Teresina. Boletim Informativo Geum. 2014;5(3):46-51.

16. Parra-Flores M, Souza-Galhardo LM, García-Correa GA, Centellas-Hinojosa S. Incidence of catheter-related infection incidence and risk factors in patients on total parenteral nutrition in a third level hospital. Cir Cir. 2016;85(2):104-8.

17. Sugrue D, Jarrell AS, Kruer R, Davis S, Johnson D, Tsui E, et al. Appropriateness of peripheral parenteral nutrition use in adult patients at an academic medical center. Clin Nutr ESPEN. 2018;23:117-21.

18. McMahon MM, Nystrom E, Braunschwerg C, Miles J, Compher C; American Society for Parenteral and Enteral Nutrition (A.S.P.E.N.) Board of Directors; American Society for Parenteral and Enteral Nutrition. A.S.P.E.N. clinical guidelines: nutrition support of adult patients with hyperglycemia. JPEN J Parenter Enteral Nutr. 2013;37(1):23-36.

19. Study Group of Hyperglycemia in Parenteral Nutrition Area of the Spanish Society of Endocrinology and Nutrition Seen; Olveira G, Tapia MJ, Ocón J, Cabrejas-Gómez C, Ballesteros-Pomar MD, Vidal-Casariego A; et al. Prevalence of diabetes, prediabetes, and stress hyperglycemia: insulin therapy and metabolic control in patients on total parenteral nutrition (prospective multicenter study). Endocr Pract. 2015;21(1):59-67.

20. Olveira G, Tapia MJ, Ocón J, Cabrejas-Gómez C, BallesterosPomar MD, Vidal-Casariego A, et al; Study Group of Hyperglycemia in Parenteral Nutrition: Nutrition Area of the Spanish Society of Endocrinology and Nutrition (SEEN). Parenteral nutrition-associated hyperglycemia in non-critically ill inpatients increases the risk of in-hospital mortality (multicenter study). Diabetes Care. 2013;36(5):1061-6.

21. Pantoja F, Fragkos KC, Patel PS, Keane N, Samaan MA, Barnova I, et al. Refeeding syndrome in adults receiving total parenteral nutrition: An audit of practice at a tertiary UK centre. Clin Nutr. 2019;38(3):1457-63.

22. Ocón-Bretón MJ, Ilundain Gonzalez AI, Altemir Trallero J, Agudo Tabuenca A, Gimeno Orna JA. Predictive factors of hypertriglyceridemia in inhospital patients during total parenteral nutrition. Nutr Hosp. 2017;34(3):505-11.

23. Lakananurak N, Tienchai K. Incidence and risk factors of parenteral nutrition-associated liver disease in hospitalized adults: A prospective cohort study. Clin Nutr ESPEN. 2019;34:81-6.

24. Llop-Talaveron J, Badia-Tahull MB, Lozano-Andreu T, SuarezLledo A, Leiva-Badosa E. Risk factors of hepatic function alterations in hospitalized adult patients treated with short-term parenteral nutrition receiving the same lipid composition at the same dose. Lipids Health Dis. 2018;17(1):267.

25. International Life Sciences Institute. 10 anos de indicadores de qualidade em terapia nutricional no Brasil/ILSI Brasil. $3^{\mathrm{a}}$ ed. São Paulo: International Life Sciences Institute; 2018.

Local de realização do estudo: Hospital Regional de Taguatinga, Taguatinga, DF, Brasil.

Conflito de interesse: Os autores declaram não haver. 